\title{
Abstracts of the 10th International Aphasia Rehabilitation Conference 24-26 July, 2002, Brisbane
}

\section{KEYNOTE PRESENTATION 1}

\section{Aphasia and Learning: A Contemporary Overview}

Audrey Holland

Department of Speech and Hearing Sciences, The University of Arizona, USA

\begin{abstract}
Tntervention in aphasia is largely a matter of skilled application of learning principles to guide and assist in recovery. This is true for counselling as well as for more direct forms of intervention. It is the contention of this paper that our understanding of the learning process is both incomplete and outdated. The purpose of this talk will be to explore recent developments in cognitive psychology that are currently having an influence on intervention, or have potential for doing so in the future. Three topics will be discussed: Connectionist theories, skills development theories, and advances in adult learning. Clinical examples will be presented as well.
\end{abstract}

\section{KEYNOTE PRESENTATION 2}

\section{The Remediation of Word-finding Impairments in Aphasia}

Lyndsey Nickels

Macquarie Centre for Cognitive Science, Macquarie University, Australia

Tmpairments of word-retrieval and production are a common and distressIing feature of aphasia, and much clinical time is devoted to attempts at their remediation. There are now many research papers devoted to case studies examining treatments for word retrieval impairments using a wide range of tasks with individuals with varying levels of impairment.

This paper will derive and discuss several main themes from the literature, these will include the contrasts between strategic approaches and facilitative or repair approaches to remediation, semantic and phonological tasks in therapy, generalisation in therapy tasks and the relationship between impairment, therapy task and outcome. Further discussion will be made regarding the relationship between impairment level treatments and measures of disability and handicap, and between therapy research and therapy practice. The paper concludes that there can be no doubt that therapy for word retrieval impairments can be highly successful, resulting in long term improvements which can be of great communicative significance for the individual with aphasia. However, predicting the precise result of a specific treatment task with a specific individual with certainty is still not possible. For clinicians the recommendation is to use analyses of functional impairments to guide the choice of task, but to ensure that efficacy is tested and not assumed. Furthermore, structured multi-modal and multicomponent 
tasks (e.g., "semantic" or "phonological" cueing hierarchies) may hold the most promise for many individuals. For researchers, there remains a need to further dissect tasks, impairments and their interactions across series of single cases.

\title{
KEYNOTE PRESENTATION 3
}

\section{Aphasia Groups: Healing Through Community}

Roberta J. Elman

Aphasia Center of California, USA

\begin{abstract}
A phasia isolates. With reduced communication skills, it is common for people with aphasia and their friends and colleagues to withdraw from one another. Research from a variety of disciplines will be presented to support the argument that social connection and community are vital for maintaining positive health and overall longevity. This research shows that creating and maintaining meaningful ties to other people is essential for being happy and healthy. Aphasia groups offer an excellent way to foster such interpersonal relationships and meaningful ties. Groups have the power to heal as shared interests, stories and trust develop among group members. The focus of the aphasia groups at the Aphasia Center of California is on moving people forward in life rather than on fixing or curing language deficits. Programs at the Aphasia Center are developed using a life participation approach within a social model of communication. Our aphasia groups provide people with membership in a community while giving them a reason or purpose for communication. Rather than being excluded from a range of activities, aphasia groups can be inclusive: everyone with aphasia belongs and everyone with aphasia is welcomed. In addition, our research demonstrates that aphasia groups can provide members with the needed support and confidence for reintegrating into the community at large.
\end{abstract}

\section{KEYNOTE PRESENTATION 4}

\section{Working Together: Conditions and Competence in Developing Aphasia Services}

Carole Pound, Jayne Lindsay and Tom Penman

Connect - The Communication Disability Network, UK

$\mathrm{W}$ ithin current health and social care policy there is a strong drive towards integrating the voice and the expertise of service users in developing and delivering services. Whilst many service planners and deliverers willingly sign up to principles of inclusion and empowerment, the practicalities of authentic collaborations often prove more elusive. This is particularly the case where aphasia highlights the complexities and subtleties of negotiation and joint working.

As a values based organisation, staff at Connect are encouraged to work creatively to ensure maximum participation by people with aphasia in developing and delivering services within the therapy, education and research programs. This paper will report on a series of initiatives to work more collaboratively with people with aphasia. These include consultation days, working parties and interest based therapy groups - for example, a photography group, an art group. By reflecting with people with aphasia on the content 
and process of these initiatives, we are gradually evolving a sounder understanding of the practicalities and challenges of working in partnership.

A cornerstone of this work has been the identification of conditions and competencies relating to participation and communication access. Drawing on two specific examples - consultation surrounding the development of a new centre in Bristol, and a co-facilitated conversation group at Connect London - we will discuss emerging themes in terms of their implications for resources and practices. We will also make recommendations for the training and support of students, volunteers, staff and people with aphasia in working to achieve more user centred services.

\section{KEYNOTE PRESENTATION 5}

\section{TBI Rehabilitation: The Road Less Travelled}

Leanne Togher

School of Communication Sciences and Disorders, Faculty of Health Sciences, The University of Sydney, Australia

R ehabilitation for people with a traumatic brain injury (TBI) has changed Rignificantly over the last 25 years. In fact, there were virtually no specialist brain injury units in Australia before the mid-1970s. Such units were established with the recognition that this group of patients had different problems and rehabilitation needs to the more typical elderly neurological population. Early clinicians and researchers attempted to conceptualise the unique communication problems that may follow TBI by extrapolating from models of aphasia rehabilitation. This focal deficit approach was superseded by a recognition of the interplay between cognition, executive functioning and so-called "higher level" language functions which frequently break down following TBI. The emergence of the "cognitive-communication" disorder has resulted in a proliferation of workbooks, computer programs and handbooks aiming to improve these skills in people with TBI. Such therapy resources are a popular inclusion in speech pathology clinics both in Australia and internationally and represent the preferred route of therapy for many time-challenged clinicians. The question remains as to how such programs affect these individuals in their everyday lives.

The goal of rehabilitating people with TBI is to promote their communicative opportunities in real life settings. It is imperative that we as clinicians provide such opportunities, however to achieve this objective we may need to take the road less travelled. Measuring communication in everyday settings remains one of the challenges facing clinicians who work with people with TBI and their social networks. The search for detailed, reliable, valid measures of everyday communication is crucial to satisfying the needs of consumers, funding agencies and speech pathologists.

Sociolinguistics provides us with a way of meeting this dilemma. Systemic Functional Linguistic (SFL) theory (Halliday, 1994) used in combination with our knowledge of the cognitive-communication problems of people with TBI can provide a powerful combination for innovative therapy design. SFL has a multi-layered perspective incorporating the interdependence of the context of the situation (e.g., field, tenor and mode), the context of culture (e.g., ideology, class, gender, ethnicity, disability) and the language used by participants in an interaction. SFL has been found to be a useful way 
of measuring the communication of people with TBI (Togher, Hand \& Code, 1997a, 1997b; Togher \& Hand, 1998, 1999; Togher, 2000). The applied nature of this theory means that treatment planning is continuous with assessment.

Treatment should incorporate current ideas regarding cognitive rehabilitation, sociolinguistic measurement and functional perspectives. Within this framework, the clinician also needs to take account of the available evidence base. PsychBITETM is a new database which will assist clinicians to make their treatment decisions using the evidence base of RCTs, patient group comparisons and single case designs (Researcher team: Tate, Perdices, Mcdonald. Togher \& Moseley). PsychBITE ${ }^{\mathrm{TM}}$ stands for the Psychological database for Brain Injury Treatment Efficacy. The database will include each clinical trial's bibliographic details, methodological design, abstract and ratings of methodological quality. To ensure reliability, two independent reviewers will eventually rate all methodological ratings listed on PsychBITETM. We are planning for PsychBITE ${ }^{\text {TM }}$ to be made available on the worldwideweb free of charge.

The future of TBI rehabilitation is exciting. It is hoped that it will consist of new initiatives such as empirically based communication rehabilitation programs, automatic computer analysis of spoken texts, improved access for rural and remote communities through telerehabilitation, psychometrically sound outcome measures, refinement of single case methodology so that it can be incorporated readily into clinical practice and finally, that appropriate rehabilitation efforts being directed to culturally and linguistically diverse populations, including Aboriginal and Torres Strait Islander people.

Finally, there are a number of larger developments in the area of TBI rehabilitation such as the use of pharmacological treatments, behavioural treatments designed to stimulate dendritic growth and advances such as intracerebral grafting. As communication specialists we are well placed to contribute to such advances in knowledge. Ultimately, however, our goal is to develop well-designed communication activities, which tap into a range of linguistic levels and which have some reality for the person with TBI and their social network.

\section{SYMPOSIUM: VERB DEFICITS IN APHASIA}

\section{The Impact on Syntactic and Discourse Competencies}

Elizabeth Armstrong ${ }^{1}$, Roelien Bastiaanse ${ }^{2}$ and Susan Edwards ${ }^{3}$

${ }^{1}$ Macquarie University, Australia

${ }^{2}$ Groningen University, The Netherlands

${ }^{3}$ The University of Reading, UK

Verb deficits are pervasive in aphasia and have profound consequences for the aphasic speaker's ability to use well formed sentences, to convey meaning and to be an effective communicator. In this symposium we consider verb deficits from a lexical, semantic and syntactic perspective and the effect of verb deficits on communicative effectiveness. The symposium will start by a review of investigations into deficits in both production and comprehension of verbs and sentences in two contrasting types of aphasia, fluent and non-fluent. The following presentations will then detail work that has examined the effect of verb deficits on sentence production in English and other languages and how these deficits may impact on an aphasic 
speaker's ability to be an effective communicator. We aim to demonstrate that carefully targeted linguistic analysis within the syntactic, lexical or discourse domain can contribute to the rehabilitative process that a therapist and aphasic speaker might engage in.

\title{
Lexical and Syntactic Properties of Verbs
}

\author{
Susan Edwards
}

The University of Reading, UK

Tn the first presentation, we will present an over-view of the lexical, synItactic and semantic properties of verbs, including predicate argument structure, verb inflection, verb movement, the effect of verb particles and event structure. We will look at how aphasia may affect either the representation of verb properties or affect a speaker's access to, or implementation of, that lexico-syntactic knowledge. In production, the type of verb affects the structure of the sentence. It has been demonstrated that while for nonaphasic speakers, the number of potential arguments a verb takes influences processing capacity, for aphasic speakers it is the number of arguments taken that affects processing (Thompson et al., 1995; Edwards, 2000). Verb inflection is vulnerable in aphasia and deficits are found in both agrammatic and paragrammatic speech (Edwards, 2001). In agrammatic speech, the frequency of errors is related to the structure of the target sentence. So, more errors have been found in auxiliary verbs in questions than in other sentence types (Arabatzi, 2000). Verb information affects meaning as well as structure and this information can impact on comprehension in aphasia. The meaning of verbs can be changed by verb particles or prepositions. The use of a verb particle such as down is linked with the event structure of the verb phrase and, for aphasic listeners, may influence sentence comprehension (Angel, 2001). We will present data from single case and group studies to illustrate how deficits in these domains of language can be assessed using currently available clinical procedures and how such deficits may differ according to the assessment regime chosen. We will consider the conflict experienced by clinicians when weighing up the need for detailed assessments versus other demands of managing a clinical case-load.

\section{The Impact of Verb Deficits on Sentence Construction}

Roelien Bastiaanse

Groningen University, The Netherlands

The second presentation will give further examples of verb deficits, this time extending the data to include different types of aphasia and languages other than English.

When word retrieval deficits in aphasia are examined, the focus is usually on nouns assessed at the word level. It is only recently that aphasia tests have included tasks that enable the clinician to look at verb retrieval (such as the latest version of the BDAE and the Verb and Sentence Test: VAST: Bastiaanse et al., 2002). Verbs carry information as outlined in the first presentation. Nouns, at least the ones that are usually included in a naming test, refer to entities in the world. Verbs, however, express relationships, as shown, for example, by the arguments that they require and by markedness for finiteness (the relationship with the subject of the sentence and in some languages with the object as well). This presentation will focus on these 
relationships and it will be argued that verbs are difficult to retrieve for aphasic speakers, regardless of aphasia type, because of the intrinsic meaning that is contained in the verb.

Data will be presented showing that the same aspects of verbs are vulnerable in all aphasic speakers, although the deficits may arise from different underlying problems. For example: Dutch speakers with Broca's and Wernicke's aphasia have been tested to study the production of finite versus non-finite verbs (Bastiaanse \& Edwards, 2001). The results show that both aphasia types are significantly impaired in the production of finite verbs. An error analysis shows, however, that speakers with Broca's aphasia tend to produce infinitives instead of finite verbs, whereas speakers with Wernicke's aphasia produce semantic paraphasias, that is finite verbs, that have a semantic relationship with the target.

These data show the importance of examination of verb retrieval at both the word and the sentence level. At the end, a case study will be presented, to show the impact of verb retrieval deficits in spontaneous speech, thus linking to the next presentation of this symposium.

\section{The Impact of Verb Deficits on the Ability to Maintain Discourse}

Elizabeth Armstrong

Macquarie University, Australia

Tn this presentation both linguistic and functional implications of verb deficits will be considered. It will be shown that analysis using a functional grammar perspective may illuminate aspects of the deficit not revealed by a lexical or syntactic analysis.

The importance of the verb as central to the meaning of a clause has been well established. However, patterns of verb use have also been acknowledged as being of prime importance at the discourse level. Research in text linguistics suggests that different genres of text, such as recount, procedure and exposition require different kinds of verbs or process types (Halliday, 1994; Eggins \& Martin, 1997). It has been proposed that a recount, for example, is characterised predominantly by material verbs (material verbs being actions of some kind, e.g., run, make), important for describing" what happened," whereas expository discourse, involving primarily description of objects/conditions and/or events requires a predominance of relational types of processes such as the verbs be and have. In addition, various components of the macrostructure of different genres require certain pattern of verbs. As a speaker's ability to participate in a variety of genres is central to his/her functional communication, disruption to the verb system may significantly affect the speaker's access to such genres.

Data will be presented in this paper that suggest that aphasic speakers may have different semantic patterns of verbs available, compared with non-aphasic speakers, and that this in turn leads to restricted communicative abilities (Armstrong, 2001). Texts of aphasic speakers will be presented which demonstrate decreased access to particular verb types and over-reliance on others, leading to impoverished or atypical attempts at certain genres. In linking lexical-syntactic resources to genres and everyday discourse, it is proposed that assessment of functional communication is possible with a theoretical linguistic basis. Such a basis can then be used for treatment planning. Implications for treatment will be discussed. 


\title{
PAPER SESSION 1: QUALITY OF LIFE AND APHASIA
}

\section{Quality of Life with and Without Stroke: Preliminary Trends}

\author{
Katherine B. Ross ${ }^{1}$, Leonard L. LaPointe ${ }^{2}$ and Richard C. Katz \\ ${ }^{1}$ Carl T. Hayden VA Medical Center, USA \\ 2 Florida State University, USA
}

\begin{abstract}
$\mathrm{L}$ oneliness, lack of social support, and lack of control over desired everyday events are common complaints of individuals who have survived one or more strokes. Treatment of quality of life by speech-language pathologists presupposes that such complaints are significantly different than those of normally-aging adults and are attributable to language and/or communication disorders resulting from stroke. The primary objective of this study is to determine whether aphasic, right hemisphere-damaged (RHD), and non-brain-injured (NBI) adults differ significantly in perceived loneliness, enacted social support, and desired control. A secondary objective is to determine whether, within groups, significant relationships exist among prognostic variables and test performance and whether, among groups, the strengths of these relationships differ.
\end{abstract}

Three psychosocial constructs (loneliness, enacted social support, and desired control over everyday events) are compared among three groups of 28 participants each (aphasic, RHD, and NBI adults) at one point in time. All participants are given the UCLA Loneliness Scale, the Inventory of Socially Supportive Behaviors, and the Desired Control Scale. Participants with aphasia or RHD are also administered standardised assessments of poststroke impairment (the Porch Index of Communicative Ability and the Behavioral Inattention Test) and activity limitation (the Communication Activities of Daily Living - Second Edition). And, the possible influence of the following personal variables are evaluated: age, education, gender, race, handedness, marital status, employment status, depression, comorbidities, overall physical disability; and, if appropriate, time poststroke, lesion location, etiology of stroke, number of strokes, presence of apraxia of speech, and presence of paralysis or paresis. To date, 12 aphasic, 9 RHD, and 21 NBI participants have completed the protocol.

Analysis of available data indicates that aphasic individuals are significantly lonelier and receive significantly more frequent social support than do NBI adults. Within NBI and aphasic groups, the presence of depression is significantly associated with increased loneliness. Within the aphasic group, the presence of hemiparesis and an etiology of ischemic stroke are also significantly associated with increased loneliness. Within the RHD group, the participant who was widowed is significantly lonelier than the rest. Within the RHD group, gender and number of lesions are significantly associated with socially supportive behaviors: females and participants with more strokes receive less frequent social support.

Available data indicate that, among groups, there is no significant difference in control over desired everyday activities. Within the NBI group, age is significantly related with desired control; younger subjects report significantly less control over desired everyday events. Within the RHD group, the presence of depression is significantly associated with decreased control, as is an increase in the number of self-reported comorbidities.

Data collection is ongoing, and final results are not yet available. If the data indicate that the psychosocial complaints of stroke survivors are significantly 
different from those of normally-aging adults and are closely related with residual language and/or communication deficits, evidence-based assessment and rehabilitation programs could be developed and implemented by speech-language pathologists.

\title{
Quality of Life in Aphasia
}

\author{
Nadine Cooper and Odette Guy \\ Communication Pathology Department, Pretoria University, South Africa
}

$\mathrm{T}$ The aim of this study was to determine the self perceptions of people with aphasia regarding their quality of life. The research made use of a semistructured interview format to elicit information regarding quality of life experiences of the person. Open ended questions regarding communication and swallowing difficulties were presented to the participants. Three participants were accompanied by their spouses during the interview. A discourse analysis was performed on the transcribed data. Although the aphasia symptoms made the analysis difficult, several themes were found. The themes included marital difficulties, social isolation, work changes and embarrassment. Despite linguistic constraints imposed by expressive aphasia, the discourse analysis yielded valuable themes, and provided novel insights into the experience of aphasia. Discourse analysis was found to be a useful tool in assisting the therapist gain a greater understanding of the experience of aphasia with regards quality of life, despite linguisitic limitations.

\section{Should Proxies Complete Quality of Life Assessments for People with Aphasia?}

\author{
Madeline Cruice ${ }^{1,2}$ Linda Worrall $^{1}$ and Louise Hickson ${ }^{1}$ \\ ${ }^{1}$ Communication Disability in Ageing Research Unit, Department of Speech Pathology and Audiology, \\ The University of Queensland, Australia \\ ${ }^{2}$ Royal Brisbane Hospital, Australia
}

Evaluating life quality of people with aphasia raises concerns for speech Dathologists and service providers. Have people with affected comprehension and expressive language faculties adequately understood the questions? How reliable are their responses? How much does aphasia interfere with thinking and personal reflection, and in turn, how much does this influence their ability to undertake life analysis? Is it more appropriate for significant others to rate the quality of life of the person with aphasia? The literature base investigating these questions is limited, as many stroke studies have excluded people with aphasia in the first instance, as well as failed to report outcomes when proxies for people with aphasia have been used (de Haan et al., 1995; Segal \& Schall, 1994). This paper presents data on a thirty people with aphasia and their proxies, and concludes that proxies can not be used in quality of life (QOL) assessment for people with aphasia.

The study evaluated the responses of people with aphasia matched with their self-selected proxies on a number of assessments and questions pertaining to QOL. These included: the QOL questions, a global rating, life domain ratings, the Short-Form 36 Health Survey, the Dartmouth COOP Charts, and the psychological well-being scale, How I Feel About Myself. Percentage agreement and reliability correlations were used to analyse the data. Thirty people with aphasia (aged 57 to 88 years) participated in the study, and selected primarily their spouses or children as their proxies. Additional information was specifically collected from proxies to delineate characteristics 
that may separate "good" from "poor" proxies, such as the proxy relationship, support role, length of association, and frequency of current contact with the person with aphasia. The emotional status of both parties was also assessed.

Findings of this study indicated that proxies had a strong negative respondent bias, that is, they under-rated or rated aphasic participants lower on global QOL, health-related QOL and well-being assessments. The paper discusses how percentage agreement varied and the concurrence between quantitative QOL ratings and proxies' qualitative descriptive answers to questions. Finally, proxy characteristics are discussed in terms of their impact on the relationship between participants' and proxies' scores. Generally, co-residential proxies, emotionally supportive proxies, and proxies who had known the participant for a longer period of time were better proxy respondents.

\section{PAPER SESSION 2: THE WHO MODEL AND APHASIA}

\section{The Use of the International Classification of Functioning, Disability, and Health as a Framework for Evaluation and Treatment of Severe Aphasia}

Travis T. Threats

Department of Communication Sciences and Disorders, Saint Louis University, USA

$\mathrm{T}$ The objective of this presentation is twofold. One purpose is to provide an overview of the World Health Organisation's 2001 International Classification of Functioning, Disability, and Health (ICF). This classification system offers a framework that keeps some essential aspects of the 1980 International Classification of Impairments, Disabilities, and Handicaps but greatly expands its view toward functioning and disability. In addition, unlike the 1980 version, which was meant for trial purposes only, the ICF was designed to be, among other uses, a practical clinical tool with fully operational definitions that could be used for evaluations and to determine progress in therapy. The ICF has two parts: "Functioning and Disability" and "Contextual Factors". Within the "Functioning and Disability" part, there are the two components of "Body Function/Body Structure" and "Activity and Participation." Within the "Contextual Factors" part, there are the two components of "Environmental Factors" and "Personal Factors". The second purpose of the presentation is to demonstrate how this framework will be especially applicable to those patients with severe aphasia. Persons with severe aphasia often do poorly on traditional standardised tests that typically emphasise Body Function level tasks. Sometimes these persons function better in their actual environments than such tests would indicate. In addition, how severity of aphasia is determined can be influenced by an ICF framework. It is possible for persons that appear moderately impaired on standardised tests that focus on impairments at the Body Function level and yet be severely restricted in their Activity/Participation skills and/or have Environmental factors which severely affect the ability to fully participate in life. The ICF is thus a system that can look at functioning from a variety of perspectives to develop a more comprehensive picture of persons' abilities. In terms of therapy, the ICF framework can be used to determine goals for this population and a method of demonstrating progress. These goals may include ones that focus on Body Function skills, direct work on Activity/Participation skills, and/or work on influencing persons' Environmental Factors. 


\section{Enhancing Aphasia Profiling of Diverse, International Populations Using the ICF-Based RAINBO Assessment System}

Gloriajean L. Wallace

Department of Communication Sciences and Disorders, University of Cincinnati, USA

The World Health Organisation has recently finalised its International Classification of Functioning, Disability and Health (ICF), a classification system which emphasises the importance of:

1. multiple-component assessment (at the body part, activity and participation levels)

2. assessing performance under non-cued as well as cued conditions

3. minimising test bias by maximising racial/ethnic, linguistic, and cultural inclusiveness for diverse, international populations

4. environmental factors (including the impact of communication partner discourse skills) and personal (idiosyncratic) factors which may enhance or deter from the overall communication profile.

Despite the fact that we now have an exquisite classification system with great potential for enhancing communication among aphasiologists at the international level, to date, there are no published aphasia assessment instruments that are compatible with the ICF in terms of all four features noted above.

This presentation will provide an overview of the Reliable Assessment Inventory of Neuro-Behavioural Organisation (RAINBO), which is a comprehensive, culturally-inclusive, ICF-based assessment system for people with neurologically-based communication and swallowing impairments. The RAINBO is entirely compatible with the ICF in terms of:

- multiple-component assessment at the body part, activity and participation levels

- comprehensive assessment of within discipline areas

- scoring system (including scoring during uncued and cued test conditions)

- attention to inclusiveness for racially/ethnically, linguistically and culturally diverse populations (to maximise use with international populations)

- inclusion of environmental factors (including assessment of communication skills of the communication partner/s), and personal factors.

The RAINBO includes ten assessment modules, a screening assessment, and a supplemental, interdisciplinary functional outcomes/quality of life measure that allow the clinician to evaluate a wide range of areas including: cognition, language, general communication, swallowing, sensory awareness, and motor skills.

This session will describe RAINBO assessment modules that are applicable for use with people who have aphasic communication impairments (including assessment of their communication partner's communication skills). ICF "Body Part, Activity, and Participation level" profiles will be presented for people with aphasia using RAINBO assessment system clinical data to demonstrate practical applications for use of the RAINBO with diverse populations from the international community. 


\section{PAPER SESSION 3: THE INSIDER'S PERSPECTIVE}

\section{Living with Aphasia: The Impact and Communication of Self-stereotypes and Other-stereotypes}

Cindy Gallois and Jeff Pittam

Centre for Social Research in Communication, School of Psychology,

The University of Queensland, Australia

The rehabilitation process for people with aphasia requires exceptional levels of motivation and commitment by the person, others in the social environment, and health professionals. Therefore, the stereotypes about aphasia held by these people, even subtly communicated, and the extent to which they are incorporated into the person's social identity, are crucial factors to the outcomes of rehabilitation and to coping with aphasia. This is particularly true given the impact of aphasia on communication itself. There is little research on the self- and other-stereotypes around aphasia and their communication. On the other hand, there is a considerable body of research on the impact of stereotypes on communication between patients and health professionals and their long-term and short-term consequences. This work indicates that stereotype-based communication by practitioners leads to reciprocal communication by patients and to negative outcomes for self-esteem and health. There is also a parallel, but relatively independent, body of research about the management of identity by patients with acquired chronic illness or disability, which shows inconsistent results about the impact of self-stereotypes on health outcomes. Thus, this is an opportune time to integrate these research traditions in the context of living with aphasia. This paper reviews the two literatures and proposes a research agenda about the impact of communication between health professionals and patients on the acquisition and maintenance of self-stereotypes around illness, ageing, and disability, and the consequences for identity, rehabilitation, and quality of life.

\section{The Co-construction of the Discharge Process}

\section{Deborah Hersh}

Department of Speech Pathology, Flinders University of South Australia, Australia

$\mathrm{T}$ his paper addresses how speech pathologists and patients with aphasia co-construct the discharge process. It explores some of the strategies that speech pathologists use to distance themselves or "wean" patients from therapy and also some of those strategies used by people with aphasia to either continue or cease therapy. The case discussed in this presentation is based upon data collected from in-depth interviews with 30 speech pathologists, 22 people with aphasia and 16 family members as part of a doctoral study. It illustrates the complex and individualised interactions that contribute to the ways in which aphasia therapy ends and sheds light on this under-researched aspect of aphasia rehabilitation. 


\title{
PAPER SESSION 4: COGNITIVE REHABILITATION
}

\section{Towards a Comprehensive Model of Cognitive Rehabilitation}

\author{
Barbara Wilson
}

Cognition and Brian Sciences Unit, Medical Research Council, Addenbrooke's Hospital, Cambridge, UK

Cognitive rehabilitation is a field that needs a broad theoretical base incorporating frameworks, theories and models from a number of different areas. No one model or group of models is sufficient to address the complex problems facing people with cognitive problems consequent upon brain injury.

This paper considers some of the models that have influenced cognitive rehabilitation including models of cognition, assessment, recovery, behaviour, emotion, compensation and learning. An attempt is made to synthesise these different models into a comprehensive model of cognitive rehabilitation.

\section{Cognitive Rehabilitation of Individuals with Cognitive Impairment After Brain Injury: A Call for a Paradigm Shift}

\author{
Robin Hanks ${ }^{1}$ and Audrey Holland ${ }^{2}$ \\ ${ }^{1}$ Department of Rehabilitation Psychology and Neuropsychology, Rehabilitation Institute of Michigan \\ Department of Physical Medicine and Rehabilitation, Wayne State University, School of Medicine, USA \\ 2 University of Arizona, USA
}

$\mathrm{T}_{\mathrm{s}}$

he Joint Committee on Interprofessional Relationships for the American Speech, Language and Hearing Association (ASHA) and Division 40 (Neuropsychology) of the American Psychological Association (APA) has developed a statement concerning the cognitive rehabilitation for persons with brain injury. The goal of this collaborative project was to encourage theoretical consideration and empirical investigation consistent with available research data, relevant theory, clinical experience, and trends in service delivery and reimbursement. In 2001, this statement was completed and endorsed by its parent organisations, and is scheduled for publication in the Journal of Head Trauma Rehabilitation in 2002. This paper session provides an overview of the statement developed by this interprofessional committee, as well as discussion regarding the viability and future directions of cognitive rehabilitation. Specifically, this session will propose a shift in thinking regarding cognitive rehabilitation, such that a focus on contextualised cognitive rehabilitation rather than generalised retraining should be considered. The "contextualised paradigm", compatible with several theories of cognition, is focused on the individual's specific activity in a specific context, regardless of whether or not the final target of intervention is at the level of participation, activity, or underlying impairment. The latter type of rehabilitation, the more generalised approach, has been termed the "traditional paradigm" and it is associated with a theory of cognition as composed of individual components that can be discretely targeted with restorative cognitive exercises or compensatory procedures. The theoretical rationale, empirical research, and pragmatics associated with current rehabilitation practices that support this contextualised approach will be discussed. Additionally, the steps required for establishing empirical clinical validation of this approach will also be presented. 


\title{
Cognitive Task-Induced Changes in Motor Cortex Excitability
}

\author{
I. Papathanasiou', S. Filipovic, R. Whurr and M. Jjahnashahi \\ ${ }^{1}$ National Hospital for Neurology and Neurosurgery and Department of Clinical Neurology, \\ University College London, UK
}
The excitability of the motor cortex to transcranial magnetic stimulation (TMS) can be modulated by a variety of motor tasks. However, there is emerging evidence that cognitive tasks may alter motor cortex excitability as well. In this study we evaluated the effect of different movement-unre- lated but left-hemisphere attributed cognitive tasks on the excitability of the left motor cortex in a group of right-handed subjects.

Two groups of tasks were used, visual searching/matching and imaginal writing/drawing tasks. Each category of task included separate blocks with different tasks: letters, numbers, semantically easy to code (i.e. geometric) shapes, and semantically hard to code shapes. The modulation of the excitability of the left motor cortex was measured through changes of the size of the motor evoked potential (MEP) induced in the right first dorsal interossesous (FDI) muscle by TMS over the left hemisphere, and was assessed relative to a baseline resting condition, as well as relative to the changes of the MEP induced in the left FDI muscle by the TMS over the right hemisphere.

We found that the size of the MEP in hand muscles increased during visual searching/matching tasks, particularly when targets were letters or geometric shapes, and the increase was significant for the dominant hand (left hemisphere) only. No such consistent effects were seen across subjects during imaginal tasks. These results are consistent with the hypothesis of left hemisphere dominance for processing of the "categorical" or semantic relationships between objects. The results also suggest that imaginal tasks that in addition to imaginal motor activity include highly visually-loaded output may not affect motor cortex excitability.

This study provides further evidence that the performance of certain cognitive tasks can modulate the excitability of the motor cortex even in the absence of overt motor activity. Understanding the patterns of task-dependent changes in motor cortex excitability will provide insights into the organisation of functional cortical networks, and may also be useful for future development of novel approaches to rehabilitation therapy.

\section{PAPER SESSION 5: TRAUMATIC BRAIN INJURY REHABILITATION}

\section{Measuring Perceived Communication Following Traumatic Brain Injury: Implications for Rehabilitation}

Jacinta M. Douglas, Pamela C. Snow and Donna McNeill-Brown

School of Human Communication Sciences, La Trobe University, Australia

mpaired self-awareness or minimisation of deficit is a frequent conse-
quence of severe traumatic brain injury (TBI). In clinical practice,
impaired self-awareness has significant ramifications for the timing of ser-
vice delivery, the nature of therapy and goal setting. For persons with TBI,
ability to perceive their own communication problems precedes motivation
to participate in communication therapy. The aim of this paper is to describe
and compare the results of two studies in which self and close other reports 
of communication ability following severe TBI were investigated. Particular emphasis is placed on the implications of the results for rehabilitation in the early and chronic stages of recovery.

Sixteen adults with severe TBI (PTA range: 14-114 days) who were receiving rehabilitation in their first year post-injury, their relatives, and treating occupational therapists and physiotherapists participated in the first study. Participants with TBI perceived themselves to have significantly fewer problems than did their relatives and therapists $(p<.05)$. No significant difference was evident between the perceptions of relatives and therapists. For the most part, the patients reported that their communication had not changed post-injury and they reported fewer difficulties than a normative group. The main problems identified by relatives and therapists were inefficient verbal output, failure to structure discourse and message inaccuracy.

A clearly different outcome was evident for a second group for whom a minimum of 2 years had elapsed since the time of injury. In the second study, the perceptions of 20 participants with severe TBI (PTA range: 14-83 days) and their close others were compared with the self and close other perceptions of a matched control group. The close other reports of communication difficulties in the participants with TBI were significantly greater than the close other reports of the control participants $(p<.01)$. However, there was no significant difference between the self and close other reports of participants with TBI. In fact, their reports of current communication difficulties and change in communication ability post-injury were strikingly similar. Difficulties characteristic of the TBI participants' communication included impaired word finding, inappropriate communicative behaviour, difficulties following, maintaining and closing conversations, and difficulties providing sufficient information in a logical manner.

While being supported by the results of the first study, the notion that TBI speakers lack insight into their own behavioural performance is challenged by the results of the second study. The level of awareness shown by these TBI speakers and the congruency between their self and close other reports indicate that rehabilitation efforts to address communication are likely to be fruitful even at this relatively late stage post-injury. Indeed, it may well be more cost effective to hold a substantial proportion of rehabilitation funds for later intervention when individuals are living in the community and have become aware of their own communication problems. Additionally, the results of the early post-injury study indicate the need to focus on the development of awareness of negative behaviours perceived by close others as well as focusing on changing negative behaviours for which congruence across self and close other reports can be shown.

\title{
Reconstructing the Autobiographical Discourse of Patients with Closed-Head Injury: The "Formatting Memory" Program
}

\author{
Maria Pachalska ${ }^{1}$ and Bruce Duncan MacQueen ${ }^{2}$ \\ ${ }^{1}$ Department of Medical Rehabilitation, Cracow Rehabilitation Center, Cracow, Poland \\ ${ }^{2}$ Department of Neurolinguistics, Rydygier Academy of Medicine, Bydgoszcz, Poland
}

The confused narrative strategies characteristic of the discourse of patients recovering from closed-head injuries often have an adverse impact on the patient's own autobiography. Among the consequences of the patient's inability to formulate a coherent account of his/her own life (which we have 
elsewhere argued is distinguishable from amnesia per se) is the loss of identity, in many cases even the destruction of family and social ties. This complex problem involves many areas of neuropsychological investigation, but it should be clear that there is an essential linguistic aspect here on the discourse level. The authors have developed a comprehensive program of neuropsychological and neurolinguistic therapy, called "Formatting Memory," aimed at helping these patients reconstruct their own life stories from the disorganised fragments preserved in their memories.

We studied 27 TBI patients (14 men, 13 women, ranging in age from 15 to 48) under treatment at the centres represented by the authors, all with closed-head traumatic brain injuries resulting in coma lasting for at least 2 weeks. Patients with dementia or severe aphasia were excluded from the study. All the patients were administered a standard battery of neuropsychological tests (WAIS-R, WMS-R, Rivermead Memory Test, WAB), along with an additional task developed by the authors, consisting in writing an autobiography (evaluated according to specific criteria), which was used to assess the patient's discourse competence.

The baseline results indicated serious disorganisation of autobiographical memory in all these patients (autobiographies containing confabulations and other serious omissions and errors). Upon completion of the Formatting Memory program the patients showed improvement in all measured parameters, but the dynamics of improvement in the task of writing an autobiography were distinguishable from those of other tests. A significant improvement was noted in autobiographical discourse competence.

In disturbances of autobiographical memory the patient does not perceive his/her memories in the first-person-singular. By systematically working on compiling and composing an autobiography, the patients not only regain access to essential facts from their own past, but also recover "ownership" of these facts and the competence in narrative discourse required to relate them to others. The authors will discuss the significance of the problems raised here, both for neuropsychological and neurolinguistic theory and for clinical practice.

The paper will be accompanied by a 5-minute videotape presenting the "Formatting Memory" program.

\title{
POSTER SESSION 1: LINGUISTIC/IMPAIRMENT LEVEL INVESTIGATIONS
}

\section{Recovery Patterns in Aphasia Revisited}

Dorothea Weniger and Brigitte Bertoni

Department of Neurology, University of Zurich, Switzerland

\begin{abstract}
A general observation to be made when examining recovery from aphasia is that the individual language functions display a different pattern of recovery. For example, gains in comprehension are usually greater than gains in confrontation naming. Given such findings the question arises as to whether the pattern of recovery is determined by the architecture of the language processing system. The aim of the present study was to examine the extent to which the different language functions recover from the onset of aphasia until a chronic state is reached.
\end{abstract}


Included in the study are 10 aphasic patients, ranging in age from 35-65 years (average 52.8 years). Etiology is vascular in all patients, site of lesion variable but restricted to the perisylvian language areas.

Extent and pattern of recovery were assessed with the Aachen Aphasia Test (AAT; Huber et al., 1983; Miller et al., 2000). The AAT provides a profile of language performance with respect to the different language modalities and the different levels of linguistic description. The psychometric properties of the AAT allow the application of single case analysis procedures (e.g., pair wise comparisons between subtest scores). The AAT was administered to all patients during the first month of illness (acute stage), a second time 4-9 months post-onset (post-acute stage), and a third time after 12 months postonset (chronic stage). All patients received intensive language therapy following the onset of aphasia. Therapy goals and procedures were based on a cognitive model of language processing. Treatment was continued until the individual patient reached a persistent level of language performance. The treatment period varied from 6-19 months (average 14.8 months).

Three patterns of recovery may be discerned: (1) significantly higher scores on all subtests of the AAT in the post acute stage, with no further improvement in the chronic stage; (2) significantly higher scores on most subtests in the post acute stage, improvements in the chronic stage being restricted to the subtests that are associated with phonological and graphemic processing; (3) significantly higher scores on most subtests in the post acute stage, improvement in the chronic stage also extending to confrontation naming.

Irrespective of the initial degree of severity, type of aphasia, and locus of lesion improvements achieved in the post acute stage were not restricted to a particular language modality/function as in the chronic stage. The two patients displaying a selective improvement of confrontation naming in the chronic stage were given the AAT early in the post acute stage. It would seem that when a chronic stage is reached semantic processing abilities cease to recover significantly whereas cognitively less demanding processing abilities such as transcoding strategies (as involved in repetition, reading aloud, writing to dictation) may continue to improve. As Goldenberg \& Spatt (1994) have argued a disconnection between the perisylvian language area and the hippocampus interferes with explicit learning of and memory for metalinguistic knowledge - necessary for semantic processing. Transcoding skills may be acquired by procedural learning.

\section{The Representation of Homophones: Evidence from Remediation}

Britta Biedermann ${ }^{1,2}$, Gerhard Blanken ${ }^{1}$ and Lyndsey Nickels ${ }^{2}$

${ }^{1}$ Albert-Ludwigs-Universität, Freiburg, Germany

${ }^{2}$ Macquarie Centre for Cognitive Science, Macquarie University, Australia

This paper will examine the production of ambiguous words (homophones e.g., pair, pear) in aphasia. In the psycholinguistic research literature there is debate regarding the representation of homophones. Some authors (e.g., Levelt, Roelofs \& Meyer, 1999) assume homophones share one phonological form, in contrast others suggest that each member of the homophone has a separate phonological representation (e.g., Caramazza, Costa, Miozzo \& Bi, 2001). This study presents evidence from the treatment of aphasia that addresses this issue. Three single cases of three aphasics with severe anomia, one German and two English, will be presented. 
The treatment comprised intensive training of picture naming using phonological cues. It was investigated if this pure phonological training could improve naming performance; what pattern of generalisation was observed across pairs of stimuli that were homophonic, semantically related or phonologically related and the duration of maintenance of the improvement. The results of the German single case showed significant generalisation to untreated homophones, but no generalisation to untreated semantically or phonologically related stimuli. The English aphasics showed the same pattern of item-specific effects for the treated homophones, and generalisation to the untreated homophones. It is argued that this supports a single phonological representation but two lemma representations for both members of a homophone pair. The results are interpreted within a discrete model (such as Levelt et al., 1999) and an interactive model of Dell (e.g., 1990).

\title{
Naming Errors in Bilingual Aphasia: Implications for Assessment and Treatment
}

Maria Kambanaros

Speech Pathology Department, Flinders University of South Australia, Australia

\begin{abstract}
case study of a Greek-English speaking aphasic person will be presented Awith examples on video of his code-switching behaviour to illustrate the important issues and challenges for monolingual speech pathologists when diagnosing and managing individuals with bilingual aphasia with and without the assistance of interpreters.
\end{abstract}

Code-switching, is common in certain bilingual communities and serves multiple functions. Yet, bilinguals generally use only one language when speaking with monolingual individuals. Australia is a multicultural community in which nearly one third of all Australians use a language other than English on a daily basis (Whitworth \& Sjardin, 1993). One of these languages is Greek, which is spoken at home by approximately $90 \%$ of the 140,000 native Greek-Australians who form the second largest ethnic community in Australia (ABS 1999).

With a median age of 57.3 years (ABS 1999), the Greek-Australian community is ageing and is thus more susceptible to health problems, including suffering a stroke.

Aphasia in bilinguals is not often addressed in Australia or elsewhere (Roberts, 2000). Part of the reason might be that only a small number of bilingual speech pathologists are practising in countries like Australia. For instance, currently only 16 practitioners are registered with Speech Pathology Australia as being bilingual Greek-English speakers (personal communication, 2000). In addition, there are no bilingual assessment methods and no bilingual norms for most of the language tests used by speech pathologists for the Greek language.

In this paper the code and language mixing of a 58-year-old bilingual aphasic male (TK) during a picture-naming task specifically designed to assess word - finding difficulties for nouns and verbs in both Greek and English will be described.

At the time of the assessment TK was 3 months post-onset following a left fronto-parietal lobe lesion His native language was Greek, but English was his primary language since the age of 14 . He was conversationally fluent in both languages prior to the stroke and it was reported that he did not show extensive code-switching before the stroke when conversing with other 
bilingual speakers or his partner who only spoke English. TK made a lot of semantic and phonological errors when attempting to retrieve the target word. He also showed language mixing which involved:

1. responding in a language different from the language of address

2. using both English and Greek words in the same response

3. spontaneous translations

4. combining a stem from a word in one language with an affix from the other.

TK's language output and errors during naming could be easily misinterpreted by a monolingual therapist (even with interpreter support).

Cases like TK also demonstrate that aphasia can selectively disrupt access to a lexical item in one language while preserving the same item in the other language, and bilingual aphasic patients may be "forced" to code-switch to get the message across because of the linguistic impairment (Munoz et al., 1999).

\section{Impairment-based Interventions in Primary Progressive Aphasia: Theoretical and Clinical Issues}

Felicity Laurence ${ }^{1}$, Margaret Manning ${ }^{2}$ and Karen Croot $^{3}$

${ }^{1}$ Centre for Human Communication Research, Flinders University and Flinders Medical Centre, Australia

${ }^{2}$ St Margaret's Hospital, Australia, and Acacia Court Aged Care Complex, Australia

${ }^{3}$ Macquarie Centre for Cognitive Science, Macquarie University, Australia

ver 200 cases of primary progressive aphasia (PPA) have been reported

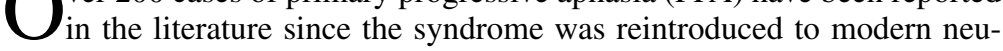
rology just 20 years ago. Of these, however, only half a dozen or so report language and communication interventions in this population. We will describe another two intervention studies designed to improve picture naming in PPA.

The first study involved CD, a 49-year-old woman who presented with a 30month history of pronoun reversal for he/she, word finding and reading and writing difficulties, and no decline in activities of daily living (ADLs). She was diagnosed with moderately severe conduction aphasia with anomia and preserved semantic-conceptual organisation. MRI and SPECT showed left temporal atrophy and hypoperfusion. Naming therapy used a crossover design with two sets of 30 line drawings matched for word frequency. CD demonstrated rapid learning but maintenance only occurred with daily rehearsal and there was no generalisation to a control set. Treatment effects returned to baseline in 5-9 weeks without rehearsal. Within 12-months of initial assessment, CD was functionally nonverbal but showed preserved associative semantics. At 22 months post-assessment, when she withdrew from therapy, CD still showed no deterioration in ADLs.

The participant in the second study was RG, a 63-year-old woman who presented with mild anomic dysphasia and progressively deteriorating reading and writing. Some non-language impairments were evident but she showed no significant memory deficits or personality change. Just over a year after presentation, following further deterioration in her language, a two phase semantic treatment was commenced to improve RG's spoken picture naming using word-picture matching tasks with semantic distractors. RG improved on treated items of high and low frequency, but spoken naming of untreated items continued to deteriorate. No generalisation to untreated items occurred. Informal assessment suggested that written naming also improved, and that 
improvement generalised to untreated items. Improvement was maintained for high frequency but not low-frequency items at two months post-treatment. RG has maintained ADLs for over 2 years since presentation and her associative semantic abilities have only recently declined.

We will compare these two studies with other published impairment-based interventions in PPA, and discuss them in relation to a theory of new word learning and forgetting in episodic memory which has been proposed to account for results in one case (Graham, Patterson, Pratt, \& Hodges, 1999).

Although the number of available studies is very small, they suggest that certain interventions might reduce anomia, with some clients showing generalisation and/or maintenance without rehearsal for periods of up to 2 months. For others, there may be no improvement in word finding, or no generalisation, or a decline in performance as soon as rehearsal is discontinued. We will discuss factors that might need consideration in management/intervention planning for this group.

\section{Aphasia Therapy on a Neurophysiological Basis: The MODAC-Concept}

Luise Lutz

Association of German Clinical Linguists, Hamburg, Germany

The MODAC-Concept (MODAC = Modality activation) was developed in the course of practical experience. It originated in the attempt to build up at least minimal linguistic and communicative skills in cases of severe aphasia and to render these skills automatic. Over the years, it has been shown that this procedure can also be effectively employed in less severe cases of aphasia.

The concept is based on considerations concerning the neurolinguistic background of aphasia which is determined by neurophysiological disturbances (i.e., disturbances of inhibitory control and of parallel processing as well as reduced cell activation).

Impairment of inhibitory processes at the synapses is found in all speech modalities and leads to various aphasic dysfunctions: insufficiently inhibited associations result in sentence blends/word blends/semantic paraphasias; mistakes in timing arise if a sound, syllable or word is not inhibited long enough.

Disturbed parallel processing leads to a variety of aphasic problems: for instance, many aphasics cannot walk and speak at the same time; while they speak one sentence they cannot plan the next one; they cannot take several grammatical decisions at the same time and, being unable to talk and listen simultaneously, they often cannot understand what they themselves are saying.

Reduced cell activation leads to difficulties in speech and comprehension: in speech, unstressed elements (unstressed syllables, function words and words that express the "themes" of an utterance) are often left out; in comprehension, unstressed elements are less well decoded than stressed elements.

The MODAC-Concept tries to reduce the problems of inhibition, parallel processing and cell activation by a series of training tasks each of which combines several language modalities. At the same time, the aim is to rebuild syntactic-semantic processing and to develop communicative abilities.

Insufficient inhibitory processes are strengthened by a) organising the exercises around 4 pictures: While the patient is dealing with one picture he has 
to suppress (inhibit) the signals from the other pictures; b) by constantly changing the tasks and modalities. Inhibitory processes that are too strong are deblocked by a) a "run-up" to the production of a word (= 7 exercises combining various modalities during which the aphasic is not asked to speak); b) using verbs as priming elements for nouns.

Reduced cell activation can be improved by a) continuously changing the modalities and tasks; b) considering the individual interests and current topics; c) experiencing success.

\section{Can Assessment Predict Facilitation Outcomes for People with Word-finding Difficulties?}

Lyndsey Nickels ${ }^{1}$, Kate Makin ${ }^{1,2}$, Belinda Crofts ${ }^{1,2}$, Melanie Moses ${ }^{1,2}$ and Christine Taylor ${ }^{1,2}$

${ }^{7}$ Macquarie Centre for Cognitive Science, Macquarie University, Australia

${ }^{2}$ Speech Pathology Service, Royal Rehabilitation Centre, Australia

T has been hypothesised that different word-finding impairments in people 1 with aphasia, will be best remediated by different treatments (e.g., Hillis \& Caramazza, 1994). To date, however, no clear or predictable relationship has emerged between the type of word-finding problem and the most appropriate treatment task (Best \& Nickels, 2000). As therapy is a time consuming and costly process, it is clearly desirable for both the client and the clinician to be able to match the nature of the word-finding problem to the most appropriate treatment, as quickly as possible.

This paper reports preliminary results from the first phase of a larger project, which aims to identify whether the response to using a task once, "facilitation", can predict the response to the same task used repeatedly over time, "therapy". The first phase, reported here examines the effects of two different tasks used once in a facilitation paradigm on subsequent picture naming of people with aphasia.

This project aims to address three questions:

- Do tasks that focus on word meaning (semantics) and those focusing on word sound (phonology) produce equal facilitation effects or do they vary in efficacy across and/or within individuals?

- Do individuals with different levels of impairment in spoken word production (e.g., semantic or phonological) respond differently to facilitation?

- Is there an interaction between $1 \& 2$ (i.e., are some tasks more effective than others for individuals with a particular level of impairment)?

To date, five individuals have been tested. Only one individual showed a significant benefit from a facilitation task, on subsequent picture naming at least 10 minutes later. This individual, benefited from phonological facilitation (repetition in the presence of a picture) but failed to benefit from semantic facilitation (feature verification, e.g., Does it bark?). No other individual showed any benefit from the facilitation tasks. Why have the results of facilitation been so limited? For phonological facilitation it is possible that retesting happens too late: some previous studies have found that there were only short term benefits from facilitation, with no lasting effects observable 10 minutes later (the time at which we retest). For semantic facilitation it is possible that the fact we do not include the phonological form of the word in the question influences efficacy: LeDorze et al. (1994) found facilitation effects from a semantic task 
ONLY when the phonological form was provided. Further investigation is currently underway, to test these hypotheses, with the individuals tested to date, and additional aphasic participants.

\title{
POSTER SESSION 2: CASE STUDIES
}

\section{A Social Approach: Solution Focused Aphasia Therapy}

\author{
Larry Boles and Mimi Lewis
}

California State University, USA

Tn previous work (Boles \& Lewis, 2000), a method of counselling couples 1 with aphasia was presented. The current study used principles of solutionfocused therapy during communication therapy for an individual with aphasia, while incorporating his wife in the conversation-based approach. Exceptions to problem behaviours were emphasised, with success based on client-generated goals. While the couple in this study conversed, the clinician encouraged certain communicative acts while discouraging others. SFAT had a "counselling" orientation, although communication improvement was the underlying goal. For example, "Louise" complained they seldom talked about "deeper issues" compared to pre-stroke conversations. In focusing on exceptions, the clinician might have asked, "when was the last time you did talk about one of these issues?" The therapy task soon became focused on how they could recreate that calmness that was revealed in Louise's response to the question. SFAT used scaled questions as the benchmark for improvement. For example, when Louise said that "Mark" rarely asked her any questions, the clinician asked Mark how difficult (1-10) it was to ask her questions. The final component of therapy was 10 minute daily conversations, during which distractions were minimised. No topics were imposed. This homework provided a safe environment in which to practice their improving skills in talking to one another. The authors of this paper challenge the following assumptions of traditional aphasia therapy:

- Once the patient has been cued, and produces the desired (by the expert) response, and that has been reinforced, that response is more likely to recur.

- The cueing used by the aphasiologist is likely to occur outside the therapy room.

- The "expert/patient" format of traditional aphasia therapy, wherein the aphasiologist elicits responses and the patient responds, is likely to generalise to the communicative situations practiced outside the therapy room.

The following principles (Simmons-Mackie, 1998) were applied to the SFAT approach:

- Conversation is the focus, rather than discrete linguistic units.

- Social interaction and information exchange are both worthy goals for therapy.

- Address communication within authentic, relevant, natural contexts.

- View communication as a dynamic, flexible, multidimensional activity.

- The collaborative nature of communication is the focal point, rather than on the individual with aphasia.

- Focus on the social and personal consequences of aphasia.

- Focus on adaptations to impairment. 
Mark and Louise made measurable gains in their communication with one another. The measures of that success included an increase in their self-ratings of communication, increased facilitative gestures by Louise, and an increase in communication independence by Mark.

\section{Legal Decision Making Capacity and Aphasia: Reflections on a Case}

Alison Ferguson', Linda Worrall, John McPhee, Rhonda Buskell, Elizabeth Armstrong and Leanne Togher

${ }^{1}$ School of Language and Media, The University of Newcastle, Australia

The decision-making capacities of interest to the present research are those that involve the exercise of the rights and responsibilities encountered in everyday citizenship. For example, decision making in the management of financial/legal affairs and with reference to life decisions (including accommodation, health decisions such as consent to intervention, advance directives or "living wills", consent to participate in health research).

Previous literature has presented anecdotal case reports, and brief reports of legal cases in which the legal competence of people with aphasia has been at issue, but there is only one study located which reported brief preliminary empirical data. These reports suggest that people with aphasia may be at risk of being considered less competent than they are to manage their affairs. On the other hand, it has been suggested that people with aphasia are also at risk of being considered more competent than they are. Some have suggested that intact general cognitive function cannot be assumed when considering whether someone with aphasia has decision-making capacity, but must be subject to assessment. However, guidelines for assessment are lacking, and it can be questioned whether reduced nonverbal performance is an artefact of the language loading on nonverbal tasks, or whether it reflects significant cognitive impairment.

What then are the bases upon which current legal and medical determinations of legal capacity are reached? This paper presents a qualitative analysis of a recent legal case in which the testamentary capacity of a woman with severe receptive and expressive dysphasia was unsuccessfully challenged. The findings with regard to the evidence documented by the court to be of key relevance are described and the qualitative methodology proposed for further research into this area will be outlined in order to invite comment and ideas.

\section{Return to Work After Aphasia Caused by a Gunshot Wound: A Case Study}

Odette Guy

Communication Pathology Department, Pretoria University, South Africa

The case study examines the year-long speech-language therapy and 1 management of a 42-year-old woman who was shot in the head. Subsequent to the gunshot wound, she presented with expressive aphasia. She was under pressure to return to work, as this was the single remaining requirement for her to regain custody of her children, and to regain her legal status. The case management is discussed, as well as the special considerations surrounding the management of gunshot wound injuries. The implications of gunshot wounds are discussed in the South African context. 


\title{
Can Rhythm Therapy Improve Language, Communication and Quality of Life for People with Aphasia?
}

Marika Schütz

Department of Logopedics and Phoniatrics, Göteborg University, Sweden

\begin{abstract}
The aim of this study was to explore whether rhythm therapy has a positive effect on language, communication and quality of life for people with aphasia. The purpose of this group therapy, a new training concept, is to stimulate the brain by a combination of colours, symbols, words, sounds and movements to music and rhythm. In Göteborg the musician and rhythm therapist Ole Moe has led rhythm therapy groups for stroke patients since 1999. The courses have been very popular and people tend to continue more than one year. Previous studies have shown improved motor function and improved verbal flow following this treatment procedure.

A group of eight people suffering from aphasia for more than one year participated in the present study. They attended a 10-week course in Rhythm Therapy. They were tested before and after therapy. Language skills, communication effectiveness and quality of life were assessed. Results of the study showed significant improvement in fluency according to the Reinvang aphasia battery (similar to Western Aphasia Battery). No statistically significant changes in communicative effectiveness were found. The results of the interviews and expressed wish of the participants to continue the program suggest that this therapy can be psychosocially stimulating for people suffering from aphasia.
\end{abstract}

\section{Second Look: Specific Treatment Strategy for Dyslexia}

Nada Žemva

Institute for Rehabilitation, Republic of Slovenia

cquired reading disorders or dyslexia has long been recognised as a significant part of the total picture of aphasia. The knowledge of types of dyslexia is useful in assessment and treatment. It provides a theoretical framework within which the therapist may form a test hypotheses about the nature and the level of breakdown, and generate logical treatment strategies.

The aim of the study was to establish oral reading. We hypothesised that individuals combine information from the lexical and non-lexical routes to read aloud. The initial process of normal reading begins with visual identification of individual letters in words and their position within the word. The patient needs to rely on visual analysis system and visual memory. This means that disrupted short-term memory can also reduce ability of oral reading.

First, we introduced strategy to the patients. We presented them a specific set of single words. They had to read them aloud. When they made a mistake, we asked them to take a short break and come back to the same word. We insisted that the patient made a real pause, before he made the second look at the same word.

We took a set of short sentences and asked the patients to read them aloud. They had to use the same strategy as in stage 1. After they performed these tasks without mistakes, we asked them to read aloud a short written paragraph. 
The effectiveness of therapy was measured by the number of words read correctly, independence in using strategy and ability to use strategy spontaneously.

We found, that the patients very often needed stimulation to make a real break. They had difficulties to look away from the word, which they read incorrectly, before they made second look.

Reading ability increased, when the patient learned to use this strategy spontaneously. Self confidence of reading abilities increased. Improved oral reading helped the patient to get additional opportunities for stimulating oral language.

The treatment resulted in progress of oral reading in different ways. We measured the number of correct responses for each patient. After treatment oral reading gradually improved. Discussion, analysis of responses, feedback about success and nature of errors all served to strengthen independence in use of strategy. Improvement in oral reading was better in those patients who learned to use strategy spontaneously.

Our study showed that the specific treatment strategy of second look can serve as alternative tool to improve the patient's reading. The therapy is easy to perform and produces rapid rewards for both the patient and the therapist.

\title{
POSTER SESSION 3: INSIDER PERSPECTIVE IN APHASIA
}

\section{Narrative Research and Aphasia: What is the Story?}

\author{
Maxine Bevin, Kerry Chamberlain and Christine Stephens
}

School of Psychology, Massey University, New Zealand

$T^{1}$ The social sciences are seeing a "textual turn" with increased interest in interpretative work such as narrative studies. Why such an interest in narrative? Narrating is a very human activity and it is suggested that narratives produce the form and meaning that is human existence. We can discover how people deal with experience by constructing stories and listening to stories. The task of narrative research is to explore the different stories - stories that give us insight to experiences and insight into the identity of the storyteller. An area of research interest has been the study of illness narratives. These explore the ways in which people cope with the changes subsequent to disease or disability. People experiencing loss, change or traumatic life events search for meaning and begin to construct explanations for the illness or disease and establish its legitimacy in their lives. Currently, our understanding of aphasia is based on what people with aphasia are able to do with their communication skills. However, understanding the experience of aphasia and how people with aphasia negotiate their world is important to the response to therapy. Parallel to the increasing interest in chronic illness and disability has been the awareness of the insider's perspective - the experience, values, priorities and expectations of people with chronic illness or disability. This has influenced research approaches with a call for research to no longer be carried out in isolation from people with disability themselves. This paper will summarise the area of narrative research and identify its potential contribution to aphasia research. It will include the discussion of a research project that explored the use of a narrative approach with three people with aphasia. Although aphasia affected the ability of these individuals to narrate their stories, they were able to construct their own accounts. The relevance of the project and 
the implications of narrative research for both clinical practice and further research will be discussed. A secondary story to emerge is the researcher's account of the sense making process.

\title{
A Comparison of Clinical Assessment and Interviews with Significant Others Using the Communication Activities of Daily Living - Second Edition (CADL-2)
}

\author{
Jillian Sellars, Bronwyn Davidson and Linda Worrall \\ Communication Disability in Ageing Research Unit, Department of Speech Pathology and Audiology, \\ The University of Queensland, Australia
}

This study sought to compare ratings of communication activity in people
with aphasia following stroke based on performance on a clinical test
and an interview with a significant other. Sixteen adults with aphasia were
administered the Communication Activities of Daily Living-Second Edition
(CADL-2). The CADL-2 is a clinical assessment that uses simulated activ-
ities to elicit communicative behaviour. The CADL-2 uses a core set of
context dependent items to elicit a variety of speech acts and verbal inter-
changes. Some of the test items also assess functional reading, writing and
mathematical abilities. An interview, based on the items from the CADL-2,
was developed for the purpose of the study and was conducted with the
aphasic participants" significant others. A comparison of the results obtained through the CADL-2 test and interview revealed no significant difference between overall scores. More detailed analysis revealed a positive relationship between overall levels of agreement and severity of the language impairment of aphasic participants. Poorer levels of agreement were found for participants with more severe language impairment. Levels of agreement were found to vary across items with some items having high agreement (e.g., choosing the appropriate bathroom to go to - male/female) and others having low agreement (e.g., recognising the meaning of a common expression, "He hit the roof"). Factors that were suggested to influence levels of agreement included the relevance of an activity to an individual's daily life, cultural and situational variables, activity complexity and the degree to which an activity involved conversational discourse.

The use of an interview with a significant other was found to have advantages over the use of a clinical test. These advantages included the ability to gain insight into the specific communicative needs of aphasic individuals and their significant others, and the ability to gain knowledge about the communication strategies employed by a person with aphasia in the course of their daily life. The results suggested that the CADL-2 test and interview could be used interchangeably in assessing communication activity in adults with aphasia following stroke, however, the complementary use of both methods is recommended. The findings of the study have relevance for the documentation of functional status, therapy planning at the activity level and the measurement of functional outcomes for people with aphasia. 


\title{
What Strategies Assist Communication with Aphasic Patients in Acute Care? Hospital Staff Perceptions Versus Speech Pathology Recommendations
}

\author{
Alison Winkworth ${ }^{1,2}$, Annette Collins ${ }^{1}$, Marcelee Gellatly ${ }^{1}$ and Bronwyn Davidson ${ }^{1,2}$ \\ ${ }^{1}$ Speech Pathology Department, Royal Brisbane Hospital \\ ${ }^{2}$ Department of Speech Pathology and Audiology, The University of Queensland, Australia
}

\begin{abstract}
$\mathrm{O}$ ne of the key objectives in the Speech Pathology Department of the Royal Brisbane Hospital is to develop simple, practical recommendations that anyone can use to immediately assist communication with patients who have recognised difficulties. In this project we were concerned with assisting hospital staff to communicate with inpatients in a dedicated acute care Stroke Unit: but do staff use the strategies we recommend?
\end{abstract}

Which strategies did hospital staff consider made the most difference, in improving not only their actual communication but also in increasing their communication attempts? In effect, how useful are our recommendations for communication with aphasic patients in hospital? Speech pathologists may have different ideas about the potential usefulness of a particular communication strategy, compared to hospital staff who are using them all day with patients.

This paper describes the results of a 3-part investigation process of the range and type of communication strategies recommended by speech pathologists and those considered most useful by hospital staff. First we tallied and categorised all the strategies the speech pathologists recommended for use with aphasic patients over a 4-month period, based on a review of medical files and patient care plans. Secondly, we surveyed hospital staff about the usefulness of the strategies, and thirdly we conducted focus groups of key staff to provide further insight into the communication dynamics between all parties, including ourselves. Along the way we discovered the importance of reflecting on our practice and evaluating our assumptions.

Findings pointed to a core group of strategies that made up the stock of those routinely recommended for communicating with most patients, while other additional recommendations emerged according to individuals' needs. Further however, the same 3 or 4 strategies seemed to be more frequently recommended than others, representing perhaps a trifecta of winning tips. For example, "use gesture" appeared to be one of the most frequently recommended to assist communication. On the other hand though, based on our ward observations this particular strategy did not appear to be used with the same frequency. Survey results will be presented to illuminate the staff rankings of strategies.

These results indicated that we needed to do more to encourage the implementation of basic communication strategies by hospital staff in their interactions with their aphasic patients. As a result of feedback we have changed our practice: we are developing new guidelines for wording and presentation of our recommendations to maximise their use. We believe that these new practices will lead to a greater recognition of the social functions of communication in the hospital environment, that in turn will mean increased opportunities for patient interaction and improved communication outcomes before discharge from the acute setting. 


\title{
Recommended Strategies for Facilitating Communication with People with Dementia: Do Spouses Use Them, Do They Know They Use Them, and How Easy is it to Know Whether They Help?
}

\author{
Taryn Kessler ${ }^{1}$, Karen Croot $^{2}$ and Leanne Togher ${ }^{1}$ \\ ${ }^{\prime}$ School of Communication Sciences and Disorders, The University of Sydney, Australia \\ 2 Macquarie Centre for Cognitive Science, Macquarie University, Australia
}

\begin{abstract}
Tn this study, we surveyed literature available to the families and profes1 sional carers of people with Dementia of the Alzheimer Type (DAT) and compiled a list of strategies recommended for facilitating communication with people with dementia. Five men with mild DAT (Mini-Mental State Examination scores of 18-23) were video- and audio-recorded interacting with their spouses in a structured problem-solving activity in which the couples discussed possible functions of an unfamiliar object (Togher et al., in preparation). Following the problem-solving activity, we asked the spouses to indicate on a checklist which of the strategies they believed they had used/not used during the interaction and compared their responses with their actual behaviours as evident from the recordings. In the final part of the study, we used the conversation analysis protocol described by Watson et al. (1999) to determine whether a number of the recommended verbal strategies were effective in repairing communication breakdown.
\end{abstract}

All spouses used the following recommended strategies: they showed, rather than just spoke about what they meant, they allowed their spouse time to respond, and they used appropriate intonation, eye contact, facial expression and slow and deliberate movements. When attempting to repair conversation breakdown, all spouses used yes/no questions and provided extra information when needed, and four out of five spouses repeated exactly or rephrased what they had previously said. Four out of the five also suggested words for their husband during occasions of word-finding difficulty and avoided figures of speech. A number of recommended strategies were not used, such as speaking in short simple sentences and speaking in a calm soothing voice. Spouses' awareness of their strategy use was variable. Effectiveness of some strategies were identified from the conversational repair analysis and this will be discussed.

\section{Traditional Healers and Aphasia: A South African Perspective}

Odette Guy and Mary Shibambu

Communication Pathology Department, Pretoria University, South Africa

\footnotetext{
$\mathrm{T}$ The study examined the views of traditional healers regarding the causes of aphasia and the management thereof. Traditional healers were interviewed on audiovisual cassette to facilitate the demonstration of natural medicines and treatment practices for aphasia. Translations were conducted and the data was transcribed for perusal. The beliefs and practices of the traditional healers is discussed in a cultural context. The conflict experienced by the traditional healers between "Western" intervention (speech-language therapy and doctors) and traditional beliefs are discussed. Implications for participation between speech-language therapists and traditional healers in light of the new community service laws and new traditional healer legislation are discussed.
} 


\title{
POSTER SESSION 4: ACCESSIBILITY, PROJECTS AND TECHNOLOGY
}

\section{Tackling Public Transport: Making it Easier for People with Aphasia}

\author{
Madeline Cruice ${ }^{1}$ Anthony Mackie ${ }^{2}$ Margaret Mitchell ${ }^{2}$ and Nicolé Robinson² \\ ${ }^{1}$ Department of Speech Pathology and Audiology, Royal Brisbane Hospital, Australia \\ ${ }^{2}$ Queensland University Aphasia Groups and CommClub, Australia
}

\begin{abstract}
Tssues of accessibility and disability awareness are fundamental concerns for people with aphasia, and represent an exciting direction in concept and practice in current aphasia rehabilitation. This poster describes the issues of accessibility and disability awareness for people with aphasia specific to local public transportation systems in metropolitan Brisbane. The project was designed with the people, for the people and by the people, through an endeavour which was gratefully funded by Disability Services Queensland Funding Program - Building Inclusive Communities 2000-2001, and sponsored by the Stroke Association of QLD Inc.
\end{abstract}

Aphasia can affect a person's ability to read, request and understand information from other people. This renders public transport communicative activities (such as reading timetables, confirming taxi/bus/ferry destination, calculating time and money/change, asking directions, and dealing with society's attitudes) confusing and difficult, and produces anxiety for the traveller with aphasia. A number of barriers to travelling with aphasia have been identified as: lack of community information and awareness about aphasia; inappropriate/ignorant attitudes towards people with aphasia; and problems personally "accessing" (understanding) public transport information. The project sought to identify, dismantle and circumvent these barriers at a local level for people with aphasia.

This poster documents the process from June to December 2001, in establishing the project and conducting the activities with a small working party of people with aphasia. It includes information on project goals, budget, activities, and dissemination of materials. The poster showcases the development of resources from the project (such as Power Point presentations; aphasia information cards and brochures; the Aphasia Travel Pack), as well as outcomes of the project (for example, media recognition). The project officer and members of the working party will be available at this poster to share further personal experiences of their involvement and to encourage others to take up the challenge in their local communities.

\section{Improving Access to Speech Pathology Services for Multi-lingual, Bilingual and ESL Aphasics}

Cindy Dilworth ${ }^{1}$ and Bronwyn Davidson ${ }^{2}$

${ }^{7}$ Royal Brisbane Hospital, Australia

${ }^{2}$ Royal Brisbane Hospital and The University of Queensland, Australia
A ustralia has become a multi-lingual society. The high levels of immi- gration that occurred after World War II and the wave of immigration associated with the War in Vietnam have resulted in different types of bilin- guals. Some of our bilinguals arrived in Australia as adults and have acquired English as a second language. Often their knowledge of English remains relatively incomplete and they may have poor literacy skills in both the home language and English. Immigrants who arrived in Australia during childhood and the children of immigrants are co-ordinate bilinguals - they 
often have good spoken communication in both languages but may have good literacy skills in English only.

Australia's society is also aging and in association with this multi-lingual, bilingual and ESL aphasics are increasingly likely to form part of the speech pathologists caseload. Australian speech pathologists need to plan for service provision to this patient group. This poster seeks to provide information to help speech pathologists in their planning and service provision.

The poster is divided into four sections. The first section examines the distribution within Australia of people who speak languages other than English at home, and then notes some of the Australian issues with regard to management of bilingual aphasia.

The second section reviews the relevant literature and provides information about language distribution and organisation within the brain as well as listing the major language features reported in bilingual aphasia. Formal and informal tests used in bilingual aphasia are also noted.

The third section provides information on how to choose the "treatment language" and looks at management options for treating in the home language and/or in English. The final section of the poster is devoted to resources and describes some of the readily available ESL and translation software as well as other opportunities provided through the internet. Some simple aphasia treatment materials available in various languages are also presented.

Speech pathologists need to rise to the challenge of providing equitable services to bilingual Australians. While working with multi-lingual, bilingual and ESL aphasics is challenging it fits well with the trend toward functional approaches and involvement of communication partners. Skills gained through working with this client group are likely to enhance our management of mono-lingual aphasics.

\section{A Computer Club for People with Aphasia}

Beverley Dodd ${ }^{1}$ and Pauline Owen ${ }^{2}$

${ }^{1}$ Private Speech Pathology Practitioner, Australia

${ }^{2}$ Senior Speech Pathologist, Southern Domiciliary Care and Rehabilitation Service, Australia

The computer club was established in September 2001, following a four week basic computer orientation course. The club has since been running on a weekly basis.

This poster presentation will cover the following areas: the origins of the club, the profile of the membership, how it is structured and operates, difficulties experienced, and reactions of and benefits to members. 


\section{Internet Training for People with a Traumatic Brain Injury - Outcomes and Barriers}

Jennifer Egan, Linda Worrall and Dorothea Oxenham-Schmidt

Communication Disability in Ageing Research Unit, Department of Speech Pathology and Audiology, The University of Queensland, Australia

The aim of this study was to determine whether people with traumatic 1 brain injury were able to learn the Internet in a one-to-one training situation using specialised training materials, which had been successfully trialed with people with aphasia.

Seven people with traumatic brain injury were each matched with a volunteer tutor. The seven participants with brain injury were aged between 20 years and 80 years and presented with a broad range of communication and cognitive disabilities. A convenient training venue with Internet access was arranged for each student-tutor pair who met for a total of six lessons. The training materials consisted of a tutor's manual and a student's manual and were designed using "aphasia-friendly" principles. The volunteer tutors taught 4 modules beginning with the easiest tasks (e.g., turning on the computer, connecting to the Internet and surfing the net), and progressing to more demanding tasks (e.g., going to a web site, saving a web site to Favourites and using email). Pre-test and post-test Internet skills assessments and interviews were conducted with participants with TBI. Volunteer tutors completed a post-training questionnaire. Although literacy disability was a barrier to people with a TBI using the Internet, other more significant barriers included difficulty concentrating through the training sessions, short-term memory loss and motivational factors. Despite these barriers, results indicate that with the use of specialised training materials in a oneto one training situation, it is possible for people with TBI to learn to use the Internet. In addition, people with a TBI and their carers reported positive outcomes beyond the acquisition of information technology skills.

\section{Blue Care Aphasia Project}

\section{Jann Offer}

Speech Pathologist/Allied Health Coordinator, Blue Care Suncoast Hinterland Region, Australia

$\mathrm{T}$ The recurring re-admission to Speech Pathology services of clients with aphasia and their carers triggered concerns that client needs were not being responded to. At this readmission, it was noted that whilst the client with aphasia experienced ongoing communication difficulties, both the clients and their carers reported restricted social contacts.

It was resolved to undertake a Community Needs Analysis with clients with aphasia and their carers in the North Brisbane Region. This community needs analysis would seek to identify those issues that result from living with a communication disability and further identify the nature of the services and assistance required.

An Aphasia Reference Group was established to guide the project. This reference group comprised representatives from a broad cross section of health professionals including Speech Pathology, Social Work, Recreational Therapy, Diversional Therapy and Nursing. A carer was also a member of the reference group. The health professionals were drawn from health 
organisations, both community and hospital based, within the Brisbane North and Redcliffe/Caboolture Districts.

The project took a two-pronged approach.

- Interviews were held with service providers currently attempting to support clients with aphasia and their carers.

- In-depth interviews were held with clients with aphasia and their carers. These semi-structured interviews were conducted for the purpose of identifying the availability and accessibility of existing programs and resources in the region and gaps in service provision.

The clients with Aphasia and their carers were selected by the Aphasia Reference Group to form a representative sample based on a range of factors. Eight individual clients and their carers contributed to the needs analysis. The clients presented with mild or moderate receptive skills and moderate or severe expressive skills. They represented residents of a broad cross-section of accommodation types including own home, residential/nursing home. The therapy status for the clients also varied with clients receiving active therapy, undergoing review, having been discharged or presently participating in therapy group programs.

Given the communication difficulties experienced by clients with aphasia, it was essential to employ communication strategies that would allow clients to individually express their needs. A tool was developed which provided visual supports to facilitate discussion about the range of difficulties, emotions, barriers and assistance required. The question and answer choices were adapted from research published by Parr et al. (1997) and refined after collaboration with members of the Prince Charles Hospital Aphasia Therapy Group. Questions included: What have been the difficulties for you? How have you felt about the stroke? Did you get enough help with ...? How do you cope with Aphasia? Carers were asked the same questions and often chose the visual supports to assist them in responding.

The recommendations of the community needs analysis are invaluable in directing future service planning and three clear directions are evident.

- While a range of services is potentially available, communication difficulties and poorer self-confidence restrict access. The knowledge and confidence of clients/carers to use existing services is essential.

- Currently available services require professional development opportunities for care workers about how to facilitate successful communication with people who present with speech and language impairments.

- The use of the visual support tool enabled clients with aphasia the opportunity for independent communication of their needs. This served to highlight the opportunity for the development of "Aphasia friendly" materials for use in assessment and care provision processes. This has the potential to allow the active participation of people with severe communication difficulties throughout the care process. 


\section{From Austria to Australia: A Language Therapy Experiment via Internet and Videotapes}

Jacqueline Stark and Christiane Pons

Department of Linguistics and Communication Research, Austrian Academy of Sciences, Vienna, Austria

$\mathrm{W}$

ith the ever-increasing advances in multimedia technology the possibilities for carrying out innovative therapy projects over long distances are expanding. The realisation of "how small the world is", or, has become as a result of these advances, is simply overwhelming. In this paper we describe the design and course of a language therapy project provided to a 63-year-old aphasic subject. The project was conceived in Vienna, Austria and was carried out by the aphasic's partner in Sydney, Australia. The interactions among the aphasic, the partner and the therapist provide insight not only into the therapy process itself but also into the nature of the aphasic's language processing difficulties. The ramifications of the project extend far beyond an improvement in communicative behaviour. Not only did communication improve for the aphasic and his partner, but their quality of life also showed a positive change in the course of the therapy process.

KW suffered a massive stroke two years prior to the project. He was hospitalised for 5 months where he received language therapy on a regular basis. At the start of the project $\mathrm{KW}$ was 63, with a college education. In addition to his profession as music teacher, he sang (tenor), composed various music pieces, played four instruments (piano, organ, trumpet and recorder) and conducted and organised orchestras. He was head of a church choir and produced musical performances including operettas and operas.

CT examination 16 months post onset revealed a large area of porencephaly involving much of the left hemisphere and suggested an old left middle cerebral artery territory infarct. There was a minor shift of midline structures to the left. There was no evidence of haemorrhage.

KW initially presented with severe global aphasia and right hemiparesis. Although his overall language comprehension improved over time, his verbal output consisted of three words: "yes", "no", the name of his partner as well as the filler "um". Speech and language therapy was provided during his 5-month hospital stay, but it was discontinued shortly after his discharge because of the assumed poor prognosis.

Since KW was a talented musician before he suffered his CVA, he received music therapy in his home. He was able to sing a scale using the words "yes" and "no", both ascending and descending. He was able to sing and play duets on the piano, both bass and treble, with his left hand.

After contact was established via a relative of KW's partner in Austria, the first author flew to Australia and evaluated KW's verbal communicative abilities. Since time was of the essence, the language assessment was brief and initial therapy trials resulted in an adapted form of the ELA-Syntax program. During a 16-day stay in Australia, language therapy was provided on an intensive basis: 40-hourly sessions (including a pre- and post-test session). In general, three sessions a day were conducted. The first session early in the morning, followed by a long break, the second session at noon and the third session at 5 p.m. During the breaks KW relaxed, slept, ate or watched TV. The language therapy sessions were taperecorded and also videotaped in his living room. KW's partner was sometimes present for the sessions and before the therapist flew back to Austria she trained her in the 
therapy procedure. The therapist and partner were in contact via email, letter and phone calls. The partner videotaped the sessions and sent the videotapes to the therapist along with notes regarding the therapy process. Language therapy was provided by the partner on an average of four sessions a week, when possible. The therapist viewed parts of the taped sessions and provided feedback via email and phone calls.

The main issues to be addressed in this presentation are:

1. What were the goals and structure of the language therapy program provided to KW?

2. What can we abstract from this single experiment regarding the language therapy process (Byng, 1995)?

3. What are the positive and negative aspects of the therapy experiment with KW?

4. How did the long distance interaction and feedback between the therapist and the aphasic's partner actually work out?

5. Regarding KW's verbal output, he produced "new", unexpected words in response to the picture stimuli (e.g., worked on: "going to the bathroom"; untrained: "walking to the bathroom"). What does an analysis of the structure of the relationship between the "new" untrained words and the anticipated, trained target words tell us about lexical processing?

6. What were the ramifications of the language therapy for KW's everyday life?

The language therapy experiment with KW proved successful. Already in the first therapy session KW was able to produce seven new words spontaneously and with minimal assistance. The positive outcome of the first session motivated $\mathrm{KW}$ and his partner and provided a positive departure point for the project. The successive sessions were characterised by intra- and inter-session variability in performance, but more importantly by minimal, though steady improvement in his verbal communicative abilities within the therapy session but also with a carryover to everyday life.

\section{Neurolmaging Techniques and the Recovery from Aphasia}

Willem van Steenbrugge

Department of Speech Pathology, Flinders University of South Australia, Australia

The aim of this poster is to present an overview of the emerging functional neuroimaging methods and procedures, and demonstrate their use in research on language processing and the recovery from stroke/aphasia, and their potential use in clinical decision making.

Imaging techniques are increasingly used in the pursuit of a better understanding of the functional and physiological factors underlying recovery from stroke and aphasia. Each of these techniques has inherent strengths and weaknesses. For instance, fMRIs have generally a very good spatial resolution displaying the cortical areas involved in cognitive processes such as language processing, but they have a poor temporal resolution making it difficult to provide an accurate account of the timing of these processes. However, the opposite is the case with ERPs. They have a very good temporal resolution but a poor spatial resolution.

Functional neuroimaging techniques are increasingly used in the pursuit of a better understanding of the functional and physiological factors underlying language processing and the recovery from stroke/aphasia (see 
Pizzamiglio, Galati \& Committeri, 2001 for a review) and reveal the following overall pattern:

- Studies in which no language task was used tended to show regression from diaschisis in the structurally unaffected regions of the $\mathrm{LH}$ as an explanation for recovery from aphasia

- Activation studies tended to show an increased role of the RH homologues of the language areas in the LH.

Given the dwindling clinical resources and restrictions, there is more emphasis on the necessity of making clinical resources more efficient and economical.

Information from functional neuroimaging may offer clinicians an avenue to economise and provide a better prediction of the clinical outcomes in their clients (Kendall \& Gonzalez Rothi, 2001) by:

- maximising the likelihood of identifying the more successful candidates for aphasia therapy

- improving our understanding of the physiological mechanisms of recovery and the efficacy of different treatment strategies.

APHASIA (All People Have A Say ... If Accommodated) GROUP

Gloriajean L. Wallace

Department of Communication Sciences and Disorders, University of Cincinnati, USA

This poster describes the content and effectiveness of an intensive four week (12 session) group program for people who are in the chronic stages of recovery from stroke/brain attack and aphasia, and their communication partners. The program, which is based on the World Health Organisation's International Classification of Functioning Disability and Health (ICF) and the US Healthy People 2010 models, is designed to enhance communication skills among people with aphasia and their communication partners while at the same time providing educational information to reduce the risk for future strokes. The program involves pre and post program communication assessment at the body part, activity and participation levels (including a pre-post synergistic assessment of conversational skills for the participants with aphasia and a variety of communication partners). Each group therapy session includes: individual work for the person with aphasia, individual conversational dyad work for the person with aphasia and a communication partner from their family or extended family network, group activities including a social time; and a guest speaker (who addresses a topic relating to reducing risk factors for stroke or education about rights and community resources for people with disabilities). The group makes one community social outing during the 4-week program.

Specific goals and objectives for the aphasia group include:

1. To enhance understanding about stroke/brain attack (what it is, signs, risk factors, the importance of early emergency room treatment, medical treatment, a review of challenges that result from stroke, and chronic post stroke issues).

2. To enhance understanding about aphasia (what it is and what it is not), and to provide information about Communication Enhancement Strategies (CES) that can be used to facilitate interactions among people with aphasia, their family members and other communication partners (including demonstration and supervised practice with the use of CES). 
3. To enhance the level of knowledge about supportive community resources, self empowerment, and the rights of people with aphasia for full societal participation according to the International Bill of Human Rights and the American with Disabilities Act.

4. To enhance the social and psychological support network for the participants with aphasia and their caregivers.

5. To enhance the quality of life and full societal participation for the participants with aphasia and their caregivers.

\title{
POSTER SESSION 5: DISCOURSE AND CONVERSATIONAL INTERACTION
}

\section{Information Exchange: Describing Dimensions Which Might Respond to Conversation Therapy}

\author{
Alison Ferguson \\ School of Language and Media, The University of Newcastle, Australia
}

\begin{abstract}
$\mathrm{T}$ his research developed a methodology to describe the exchange of information in interactions involving people with aphasia. The sampling involves a short (less than five minute) conversational interaction in which the person with aphasia provides an eye-witness account of a mock car accident. This sampling allows for comparable sampling across conditions, and over time. The sampling retains naturalistic validity, while at the same time being possible to administer in a clinical setting. The shortness of the sample enables quick transcription, similar in time to other frequently used discourse sampling methods such as picture description, while providing a more valid sampling of conversational interaction than monologic sampling. Analyses of information exchanged were identified from the literature and applied using currently available software (SALT). These measures include measures of quantity - Correct Information Units (per turn, per minute); measures of quality - Grammatical Intricacy; measures of efficiency utterances with pauses, with mazes; and measures of collaborativeness interrupted/overlapping utterances. These analyses provide clinicians with a set of linguistic descriptive measures which are feasible to implement in a clinical setting. Findings are presented from a study of 10 people with aphasia following left cerebrovascular accident and 20 non-aphasic control subjects whose information exchange is compared across communication partners. It is suggested that this methodology is well suited for use as part of the description of outcomes from conversation therapy, since it allows for quick, valid, reliable measurement of relevant conversational parameters of both the person with aphasia and their communication partner.
\end{abstract}

\section{Perseveration in Traumatic Brain Injury: Conversational Considerations}

Tali Rootshtain and Claire Penn

Department of Speech Pathology and Audiology, School of Human and Community Development, University of the Witwatersrand, South Africa

$\mathrm{T}$ This study investigated the manifestation of perseveration in conversation, utilising a Conversational Analysis approach, in two adults with Traumatic Brain Injury. Both participants were permanent residents of a facility caring for individuals with a variety of physical and cognitive disabilities and had resided there for several years. It was hypothesised that deficits in behavioural inhibition and resulting influence on working 
memory and other executive and self-regulatory skills could account for manifestations of perseveration in conversational discourse. A theoretically cohesive model of prefrontal cortex functioning (Barkley, 1997) was used as a framework within which to explore and interpret conversational and neuropsychological correlates of perseveration. The attribution of perseverative phenomena to disinhibition inferred the suitability of pharmacological intervention to improve attentional processes. The drug of choice was Ritalin, owing to the fact that the symptoms of inattention, distractibility, disorganisation, impulsivity and emotional lability, common to both ADHD and TBI, tend to be ameliorated with stimulant medication in low and moderate doses. The response of perseveration in conversations as well as neuropsychological functions was measured in a quasi-experimental design employing baseline, active, placebo and withdrawal phases. A double blind control measure was also employed to minimise rater and researcher bias. For both subjects, findings indicated that perseveration did not affect turn taking or repair ability but that topic management was compromised. It was further confirmed that outcomes related to topic management co-occurred with reduced capacities in the areas of behavioural inhibition, working memory and reconstitution (the ability to create novel and complex behaviour). A positive response to pharmacotherapy was noted in measures of behaviour inhibition and verbal fluency tasks (reconstitution). Qualitative improvements were observed in verbal working memory tasks. These changes corresponded to improvements in topic management. The extent of the improvement was tempered by several factors, including the severity of impairment and time since onset with specific reference to the chronic effects of institutionalisation. Analysis of the findings indicates the significance of the relationship between cognitive skills such as attention and working memory to conversational ability. Clear links were uncovered between perseveration and disruption to these cognitive processes, particularly in behavioural inhibition, as hypothesised. Theoretical implications resulting from the study clearly prescribe directions for future investigation and treatment, while clinical implications highlight specific avenues for intervention.

\section{Discourse Performance Following Right Brain Damage}

Sue Sherratt ${ }^{1}$ and Karen Bryan ${ }^{2}$

${ }^{1}$ Department of Speech Pathology, John Hunter Hospital, Newcastle, Australia

${ }^{2}$ European Institute of Health and Medical Sciences, University of Surrey, UK

The findings of previous research into the discourse performance of right brain damaged ("RBD") subjects have been conflicting; their discourse has been labelled alternatively as verbose or limited, syntactically complex or simple, cohesively adequate or reduced and hyperfluent or dysfluent. Furthermore, contradictory descriptions of their ability to formulate appropriately structured discourse have been noted. The discourse genres, tasks and topics assessed have varied widely and may be a contributory factor to the inconsistency in findings.

The aim of this study was to examine a variety of discourse tasks, methods of assessment and aspects of attention to provide a unique analysis of the effect of these aspects on the discourse performance of RBD subjects. Narrative and procedural discourse samples were orally elicited using a variety of topics and methods. These were analysed in terms of 7 aspects (viz. relevance, discourse grammar, clarity, productivity, syntactic complexity 
and clausal structure, cohesion and dysfluency). The results were correlated with the status of their general communication abilities and attentional mechanisms. Their discourse performance was also compared to a group of non-brain-damaged subjects matched for age and socio-economic status. On certain measures, the RBD subjects performed consistently less well than the control group regardless of discourse genre, method of elicitation and topic. However, on other measures, the RBD group's performance differed from the control group depending on the nature of the discourse task used.

Due to the reported heterogeneity of the RBD population and the variability observed during data analysis, the RBD subjects were investigated using a single-case study approach and three potential sub-groups were identified on the basis of the defining characteristics of their discourse performance. The findings of the discourse analysis of both the RBD group as a whole and the sub-groups are explained in terms of a multi-level model of discourse processing. Such a model can form the foundation for more accurate and appropriate assessment and management programs for the communication impairments exhibited by RBD subjects.

\section{Discourse Mechanisms in Bilingual Aphasia: Culture, Code Switching and Compensation}

Claire Penn', Lisa Kalmek', Dale Ogilvy², Analou Venter ${ }^{2}$ and Ingrid von Bentheim²

'University of the Witwatersrand, South Africa

${ }^{2}$ University of Cape Town, South Africa

Despite the fact that there is increasing linguistic and cultural diversity in clinical caseloads, there remains little research on the importance of such aspects on mechanisms of recovery in aphasia as well as a shortage of appropriate assessment paradigms. This paper will report on a series of studies conducted on the discourse of a group of bilingual persons with mild to moderate aphasia in South Africa. A series of narrative tasks provided an opportunity to examine in depth the interplay of cultural, sociolinguistic and compensatory strategies. The syntactic and coherence features of aphasic and control discourse samples will be presented and a number of code switching and stylistic aspects described. The findings have implications for language processing and pragmatic mechanisms in bilingual populations. It is suggested that persons with a strong bilingual tradition display patterns of code switching, flexible word order and stylistic intensification which provide a robust compensatory and scaffolding structure for some of the primary aphasic deficits. The genre of narrative provides a strong basis for such pragmatic strengths to emerge. The paper will consider how cultural factors play an important role in both diagnosis and therapy with aphasia. 


\title{
POSTER SESSION 6: INSIDER PERSPECTIVES IN TBI AND DEMENTIA
}

\section{Participant Perceptions of Group Language Therapy for People with Huntington Disease Living in a Residential Care Facility}

\author{
Maria Berarducci' ${ }^{1}$ Emma Power ${ }^{2}$, Alison Anderson ${ }^{2}$ and Leanne Togher ${ }^{3}$ \\ ${ }^{1}$ St Vincent's Hospital, Australia \\ ${ }^{2}$ Lottie Stewart Hospital, Australia \\ ${ }^{3}$ The University of Sydney, Australia
}

$\mathrm{T}_{1}$ he aim of this study was to evaluate a language therapy group for people living with Huntington Disease through data of their own perceptions of the group and their communication skills. We will outline our experience conducting group therapy and discuss the results of intervention specifically related to participant interaction, interest, involvement and communicative ability. Lottie Stewart Hospital operates a specialised residential care facility for people with Huntington Disease (HD). Cognitive and language difficulties have been attributed to subcortical dementia associated with HD. With up to $88 \%$ of people affected by HD requiring long-term care facilities (Nance \& Sanders, 1996) it is valuable to explore the impact of the treatment of their language difficulties in this environment.

Although there are a number of studies describing the changes of language function in $\mathrm{HD}$, there is a lack of literature describing therapeutic treatments for their language difficulties. Bourgeois (1991) reviewed the literature on communication treatments for adults with dementia (specifically the Alzheimer's type) and described four basic approaches. One of the approaches we decided to trial was group therapy intervention.

A language therapy intervention group was conducted over 5 weeks (45-60 minute session twice weekly) during November and December 2001. Six people with Huntington disease agreed to participate. A pre-group survey was conducted on the participant's perception of their communication skills and their abilities to use these in their communicative environment. This survey was repeated at the conclusion of the group. Pre and post measures of communicative interaction were obtained during a group problem-solving task. Segments of therapy were also analysed for initiation of communicative behaviours. At the conclusion of the therapy program a focus group with participants was conducted to gain their perceptions and satisfaction levels of the language therapy group.

Results of the pre and post group survey indicated that participants demonstrated difficulty reflecting on their own communication abilities. Analysis of the therapy sessions demonstrated that participants initially focussed their interactions on the group facilitator however, over time they were able to converse more with other participants. Qualitative analysis indicated that group members increased their communication attempts, more markedly with other participants rather than with the group facilitator alone. The focus group demonstrated that participants enjoyed attending the group, believed they made gains in their communication skills and would like to continue in the language group. However there were difficulties with obtaining satisfaction levels in a population that is cognitively impaired, with little speech. Nonverbal communicative intents and behaviour changes could not be obtained via questionnaire but were documented on-line.

There is a need to look toward other methods of obtaining such data. This study brings up issues surrounding our ability to measure participant 
perceptions of their difficulties in a disease where lack of insight is frequently one of its major symptoms.

\title{
Moving from Passive Recipient to Active Participant: Discourse Analysis in the Management of Dementia
}

\author{
Debbie Bethlehem and Odette Guy \\ Department of Speech and Hearing Therapy, Communication Pathology Department, \\ University of the Witwatersrand, University of Pretoria, South Africa
}

\begin{abstract}
lzheimer's Disease research has intensified over the past decade, with Amany new insights being revealed. However, even though advances in the care of dementia have been made, the person with the disease, more often than not, remains excluded from the decision making process. Few investigations consider the person's perspectives on events taking place, the experience of the disease, and how this affects their quality of life. This paper reports on the use of a qualitative technique (discourse analysis) with three individuals diagnosed with probable Alzheimer's Disease. The paper discusses the personal insight of these three individuals who were in the early stage of the disease progression at time of data collection. Implications for future involvement of people with dementia regarding their own care are discussed.
\end{abstract}

\section{Is Primary Progressive Aphasia a Unique Communication Disorder? Evidence from Discourse Analysis}

Alexia Miller and Kathryn Hird

Department of Human Communication Science, Curtin University of Technology, Australia

$\mathrm{O}$ ver the past decade, research employing advanced neuroimaging techniques has failed to provide evidence for absolute modularity of brain function and the homogeneity of aphasic disorders. As a result, recent theories posit a more dynamic approach to cognition, suggesting that deficits in attention and or working memory may underlie communication disorders secondary to a neurological impairment. Despite this paradigm shift, the applicability of the dynamic theory of cognition to the progressive neurological disorders, Alzheimer's disease (AD) and primary progressive aphasia (PPA) has been largely unexplored. Furthermore, the implications of the assumptions of this theory for the diagnosis, and clinical assessment and management of $\mathrm{AD}$ and PPA have not been considered.

Although the dynamic approach to cognition is supported by research investigating the speech and language of individuals clinically presenting with $\mathrm{AD}$, despite the overlapping symptomatology of AD and PPA, research into PPA reports anomia as the principal cognitive impairment within the first 2 years post-onset. Therefore, this study aimed to investigate whether PPA in the first 2 years post-onset is indicative of the selective impairment of lexical retrieval or similarly to $\mathrm{AD}$, deficits in the cognitive domains of working memory and attention. From this, the secondary aim of this study was to evaluate the validity of the modular and dynamic theories of cognition as a basis for interpreting the brain-behaviour relationship and classifying progressive neurological disorders.

The Cookie Theft picture description task was used to elicit discourse samples from 6 AD patients, 7 PPA patients and 10 non-brain-injured individuals. This task was chosen as the conceptualisation of a discourse macro-plan is a dynamic process that entails the integration of linguistic, pragmatic and 
cognitive (i.e., attention and working memory) resources. Three measures were used to analyse and compare participants" macro-planning abilities: (1) communicative informativeness and efficiency (Nicholas \& Brookshire, 1993); (2) global coherence (Christiansen, 1995) and; (3) self-monitoring and repair (McNamara, Obler, Au, Durso \& Albert, 1992).

An important finding of this study was that the AD and PPA participants, who were matched for age, dementia severity and time post-diagnosis, were indistinguishable on all discourse measures. When compared with the nonbrain-injured participants, the discourse produced by the PPA and AD patients was found to be less communicatively informative and efficient, less coherent and less effectively self-monitored. These findings challenge the modular paradigm by suggesting that language is not an isolated entity, but rather it is a dynamic process whose conceptualisation is subserved by numerous cognitive resources. The overlapping discourse performance profiles of the PPA and AD participants pose implications for the currently accepted diagnostic criteria of these conditions and thus challenge the validity of the modular paradigm as a basis for classifying progressive neurological disorders. The theoretical and diagnostic implications of this study highlight the need for speech pathologists to explore higher-level cognitive processes in order to identify the underlying cause of communication breakdown. Furthermore, these implications illustrate the importance of employing an individualistic approach, regardless of medical diagnosis, when making decisions about a patient's clinical management. 